\title{
Free-ordered CUG on Chemical Abstract Machine
}

\author{
Satoshi Tojo \\ Mitsubishi Research Institute, Inc. \\ (c-mail) tojo@mri.co.jp
}

\begin{abstract}
We propose a paradigm for concurrent natural language generation. [n order to represent grammar rules distributively, we adopt categorial unification grammar (CUG) where each category owns its functional type. We augment typed lambda calculus with several new combinatoss, to make the order of $\lambda$-conversions free for partial / local processing. 'The concurtent calcrilus is modeled with Chemical Abstract Machine. We show an example of a Japanese causative auxiliary verb that reguires a drastic rearrangement of case domination.
\end{abstract}

\section{Introduction}

Parallel and distributed computation is expected to be the main stream of information processing. In the conventional generation, the rules for composition are given from the outside and those rules control all the bchavior of the symbols or the objects, for assembling a hierarchical treo structure. For example, all the linguistic objects, such as words and phrases must be applied to socalled grammar rules to form grammatical structures or rational semantic representations, under a strict controller process. However, this kind of formalization obvionsly contradicts the partial / distributed processing that would be required in parallel architecture in future.

In order to represent grammar rules distributively, we adopt categorial granmas, where we can an attach local grammar rule to each word and phrase. What we aim in this paper is to propose a paradigm that enables partial / local generation through decompositions and reorganizations of tentative local structures.

In the following section, we introduce the $\mathrm{cx}$ tended $\lambda$-calculus. Thereafter we introduce the ChAM model and we reinterpret the model in terms of natural language processings. J'hon we show the model of membrane interaction model with the example of Japanese causative sentence that requires drastic change of domination of cases. Finally wo will discuss the future of the model.

\section{Extended typed $\lambda$-calculus}

CUG (Categorial Unification Grammar) [8] is advantageous, compared to other phrase structure grammars, for parallel architecture, because we can regard categories as functional types and we can represent grammar rules locally. This means that we do not need externally-given grammar rules but those rules reside within each word or each phrase. In this section, we regard categories as polymorphic types and consider the type calculus. In later sections we denote categories by D $\Lambda$ G (directed acyclic graph) of PATR grammar [5].

\section{$2.1 \lambda$-calculus of polymorphic type}

We use greck letters, for type schemas. lior type constiantis we use $\sigma, \tau, \cdots$ while for type variables we use $\alpha, \beta, \cdots a: \alpha$ represents that the object $a$ is of type $\alpha$. If $\alpha$ and $\beta$ are types, then $\alpha \rightarrow \beta$ is a type.

The purpose of type inference is to infer the type of an object from a set of objects whose typess are known. We presuppose that two type variables $\alpha$ and $\beta$ are unified with a unifier $\theta$. We nse I' for this set of type-known objects. 'The most important two rules are as follows: ${ }^{1}$

$$
\frac{L^{2} \theta_{1} \cup\left\{x: \alpha \theta_{1}\right\} \vdash t: \beta}{\mathrm{I}_{1} \vdash \frac{1}{\lambda x^{\alpha} \cdot l: \alpha \theta_{1} \rightarrow \beta}}
$$

$$
\frac{\Gamma \theta_{2} \theta_{3} \theta_{4} \vdash \iota: \alpha \theta_{4} \rightarrow \beta \theta_{4} \quad \Gamma \theta_{2} \theta_{3} \theta_{1} \vdash s: \alpha \theta_{4}}{\Gamma \theta_{2} \theta_{3} \theta_{4} \vdash l(s): \beta \theta_{4}}
$$

The rule (2) corresponds to $\beta$-conversion of the ordinary $\lambda$-calculus [4].

\subsection{Extended combinators}

In this subsection, we introduce two combinators that enable us to change the order of $\lambda$ conversion, proposed by Steedman [6], as a kind of type change [3]. The ordinary $\lambda$-calculus requires

\footnotetext{
${ }^{1} \theta_{2}, \theta_{3}$ are for l $\theta_{2} \vdash t: \alpha \rightarrow \beta$ and for l' $\theta_{3} \vdash s: \alpha$, respectively. $\theta_{4}$ unifies $\alpha$ which appears in both type declarations.
} 
a strict order of conversion. Ilowever, in a concent rent model, this kind of strict order is a hindrance and contingent conversions are required.

Cocombinator changes the order of $\lambda$ variables as follows:

$$
C(\lambda x y \cdot f(x, y))=-\lambda y x \cdot f(x, y)
$$

Another requirement for exchanges of the order of $\lambda$-conversion is the following case. Suppose that we are recuired to compose all the lollowing typed objects:

$$
\left\{\begin{array}{l}
f: \beta \cdots \gamma \\
g: \alpha \rightarrow \beta \\
a: \alpha
\end{array}\right.
$$

In such a case, wo need to concatenate $g$ and $a$ first, and then g(a) becomes applicable to f. However, with the lielp of the following $B$-combinator:

$$
\mathbf{B}(\lambda x \cdot f(x))(\lambda y \cdot g(y)): \lambda x \cdot f(g(x))
$$

The $\lambda$-variable in 9 can be shifted beyoud the scope of $f$ so that we can concatende $f$ and $g$ first, and, thus, have a becone applicablo as in l'ig. 1.

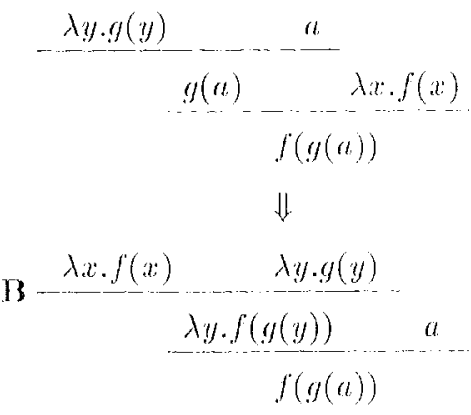

liggure 1: 13-combinator

\subsection{Cost of umification}

I'lae repealed use of $\mathrm{C}$ - and 13 -combinators is still problematic if we consider implementing it as an actual system because the termination of processing is not guaranted. We have modeded the procoss of a partial decomposition as an alsitraction of an algument of the first-oreler term. If this abstraction occurs tandomly, the process easily falls into a loop. In order to avoid this, we assume the unification cost. If a compound term (a subtreo) were to bo acomposed onse, the element with the longer distance should be abstracted first. Wo can regard the whole sentence structure an more grammatical if the sum of these nnification costs is smafler. We introduce the leeuristic costs [7], considering the parallelism between syntadic cases and semantic roles, as follows:

$$
\begin{aligned}
& \left\|\theta_{(n o m, a q t)}\right\| \cdots 1 \quad\left\|\theta_{(\omega b ;, n o m)}\right\|=k \\
& \left\|\theta_{(\text {dab,col) }}\right\|:-: 1 \quad\left\|\theta_{(i b l, a, g)}\right\|: \cdots k \\
& \left\|\theta_{(a c c, o b j)}\right\|=1 \quad\left\|\theta_{(\ell, o b j)}\right\|:-=k \\
& \left\|\theta_{(n o m, d u t)}\right\| \cdots \infty \\
& \left\|\theta_{(u, i l, c, i l)}\right\|=\infty \\
& \text { : }
\end{aligned}
$$

where $\theta_{(x, y)}$ represents a unifier of two 1$) A G$ 's: one's syntactic case is a and the other's semantic role is $y$. $k$ is some comstant larger than $1(k>1)$.

\section{Chemical Abstract Machine}

Chemical Abshact Mardhine ('hAM, for short) [1] i.s a paradigm of concentent $\lambda$-calculuss. In this pa per, we will mention our principles on natural lan-

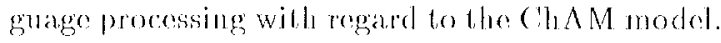

Wo assume the process of natural language? recognition as follows. Whenever a linguistic ob. ject $\mathrm{i}$ recognized, it is thrown into the solution of Ch $\triangle \mathrm{M}$, and acts as a molecule. Vorbs and some other auxiliary verbs introduces membranes. These membranes becomes their scopes for case (or role) dombation; mancly, each verb scarches for molecules (noun phrases) that aro necessary to satisfy each verb's case (role) frame, within its mentrane. In some occasions, if multiple verbs exist in one sentence, they may conflict as to which verb dominales which nomo phrase. In such a cases, two membranes can interact and call exchange sone molecules.

We $11 s^{2} s_{1}, s_{2}, s_{3}, \cdots$ for mombranes. When a mombrane si contains a molecule $\alpha$, we denote as $\psi_{i}=\alpha$. The suppoting relation (f-e) can be itllerpreted as an inclusion relation (D) in this casce. 'l'wo membranes can in benact when they conlact

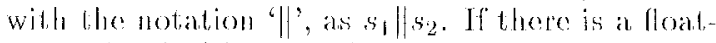
ing molecule (that which is not yot concatenated with other molecules) on one side, it can nove through the porous membranes. Valences for con catenation of each molecule are represented by typer lambele variables. If one mombrane conlaius only one composite structure, and it still bassurplus valences, we can regard that wholo the membrane has those supplus valences as follows.

$$
\begin{gathered}
s_{2} \mid=\lambda x y \text { make }(x, y, z) \\
\mid \\
\lambda x y x_{2} \mid \text { make }(x, y, z)
\end{gathered}
$$

Now, we will apply our notions above to the actura problem of sentence generation. 


\begin{tabular}{l|l} 
(yom- = read) & Who reads? \\
\hline Ken-wa Naomi-ni hon-wo yom-u. & Ken \\
Ken-wa Naomi-ni hon-wo yom-ase-ru. & Naomi \\
Ken-wa Naomi-ni hon-wo yom-are-mu. & Naomi \\
Ken-wa Naomi-ni hon-wo yom-ase-(r)-are-ru. & Ken
\end{tabular}

$$
\left(\begin{array}{ll}
-w a & \text { : nominative case marker } \\
-n i & \text { : dative case marker } \\
-w o & : \text { accusative case marker' } \\
\text { hon } & \text { : noun for 'book' } \\
\text { yom- } & \text { : root of verb 'read' } \\
- \text { ase- } & \text { : auxiliary verb for causative } \\
- \text { are- } & \text { : auxiliary verb for passive } \\
-(r) u & \text { : present tense marker }
\end{array}\right)
$$

Table 1: Agents alternation by agglutination of auxiliary verbs

\section{Example: Japanese causative sentence}

In the Japanese language, the causative and the change of voice are realized by agglutinations of those auxiliary verbs at the tail of current verbs. These anxiliary verbs as well as ordinary verbs can dominate some cases so that these agglutinations may change the whole syntax. [9]. Namely the scope of the operation of these auxiliary verbs is not the operated verb but the whole sentence. In order to illustrate these role changes, we show the alternation of the agent of the main verb in Table 1 with a short tip to Japanese lexicon.

As an example, we will take the sentence:

Ken-wa Naomi-ni hon-wo yom-aseru.

(Ken makes Naomi read tho book.)

First, we give DAG's fol each lexical items in Fig 2. The last DAG in lijg. 2 represents that the verb 'yomu (read)' requires two roles 'the reader' and 'the object to be read', and one optional role 'the counter-agent' who hears what the reader reads. In that figure, ' $W \models$ ' moans that each word is recognized in the general. world however a verb 'yomu' introduced a special membrane $s_{1}$ as a subworld of $W$. Each DAG means a polymorphic type of the lexical item.

Assume that there is a parser that constructs partial tree structures, as recognizing each word from the head sequentially. 'Then, whon the first four words are recognized, they can form a complete sentence of $(3)$.

$$
s_{1} \mid=\left\{\operatorname{read}\left(\left.K\right|_{O_{1}},\left.N\right|_{O_{2}},\left.B\right|_{\theta_{3}}\right):\left[\begin{array}{cc}
\operatorname{cat} & S
\end{array}\right]\right\}
$$

Because all the three nouns are adequately concatenatod by 'read', a sentential ropresentation is made in the subworld of $s_{1}$. In (3), $\theta_{i}$ 's are the records of unification, that contain the costs and the original types; they become necessaly when they arc backtracked, and in that meaning, those bindings are transitive.

Now, let 11 s recapitulate what has occurred in the mombrane $3_{1}$. There were four lexical items in the set, and they are duly organized to a sentence and $s_{1}$ becomos a singleton.

$$
\begin{aligned}
s_{1}= & \{K: N, N: N, B: N, \\
& \lambda x y z \operatorname{read}(x, y, z): N \rightarrow N \rightarrow N \rightarrow S\} \\
& \downarrow \\
s_{1}= & \{\operatorname{read}(K, N, B)\}
\end{aligned}
$$

Then, the problematic final word "-aseru (causative)' arrives; its J)AG representation is as in lig. 3. The $\mathrm{D} \Lambda \mathrm{G}$ in lig. 3 requires a sentential form (category $S$ ) as an argument, and in addition, it subcategorizes an item of category $N$ as an agent of the subsentence.

Now, the process becomes as in liig. 4. All through the process in Fig. 4, C- and Bcombinators are used repeatedly as well as ordinary type inference (1) and (2). The second membrane $s_{2}$ requires an agent role (the variable $x^{\prime}$ of make). 'l'here is a record in $\theta_{1}$ that it bit agenl, so that the comparison should be made between $\theta_{1}$ and $\theta_{1}\left(=\theta_{\left(K, x^{\prime}\right)}\right)$. Ilowever, because both of $\theta_{1}$ and $\theta_{1}$ unifies nominative case and agent role, the costs are equivalent. In such a case, linguistic heuristics will solve the problem. In this case, the agent of make should be the nominative of the whole sentence, and the co-agent of make is the dative of the whole sentence, so that $K$ and $N$ are bit by newly arrived make. $B$ remains bound to read, becallse there is no $\lambda$-variable of that type in make. Tho process is depicted in fig. 5 . 


$$
\begin{aligned}
& W \models K(=K e n-w a):\left[\begin{array}{ll}
\text { cat } & N \\
\text { case } & \text { nom }
\end{array}\right] \\
& W \models N\left(=\text { Naomi-ni) }:\left[\begin{array}{ll}
c a l & N \\
\text { case } & d a t
\end{array}\right]\right. \\
& W \mid=B(=h o n-w o):\left[\begin{array}{ll}
\text { cat } & N \\
\text { case } & \text { acc }
\end{array}\right]
\end{aligned}
$$

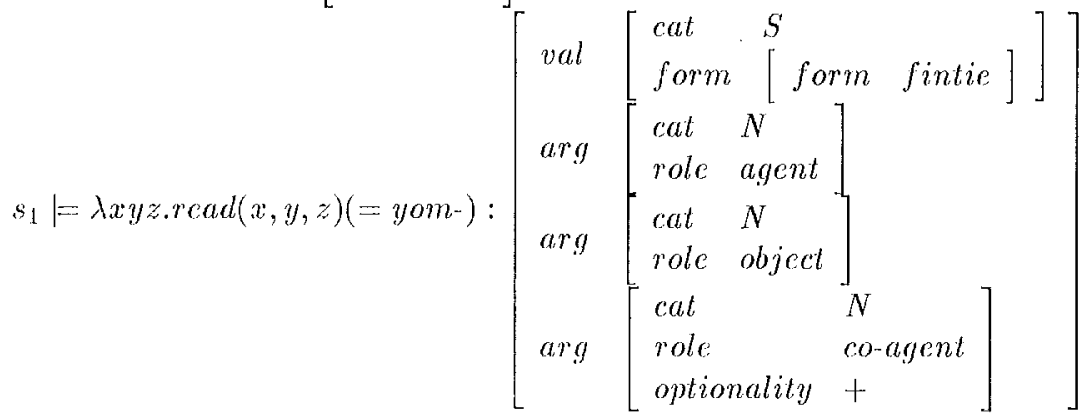

l'igure 2: Initial D $\Lambda \mathrm{G}$

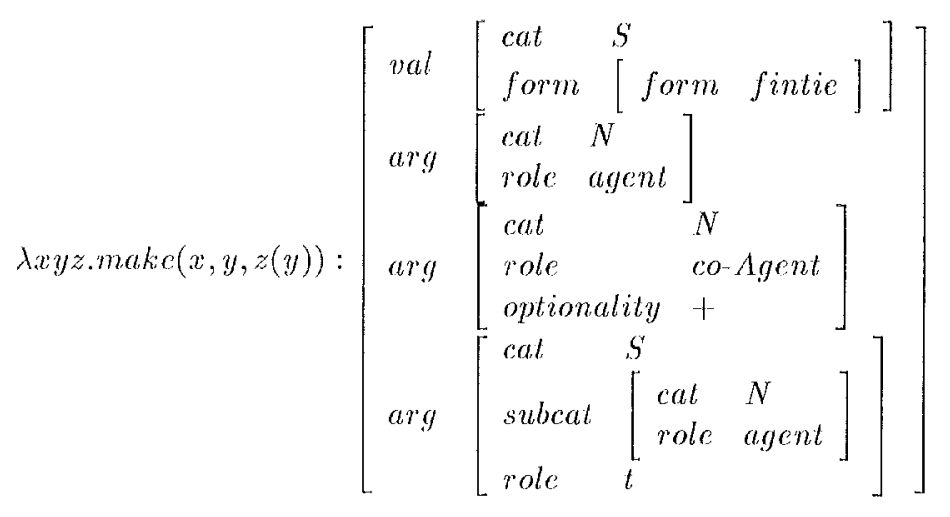

Figure 3: ])AG for 'make'

$$
\begin{aligned}
& s_{1}=\operatorname{read}\left(\left.K\right|_{\theta_{1}},\left.N\right|_{\theta_{2}},\left.B\right|_{\theta_{3}}\right) \quad \| \lambda x^{\prime} y^{\prime} z^{\prime} \cdot s_{2} \mid=\operatorname{mak}\left(x^{\prime}, y^{\prime}, z^{\prime}\right) \\
& \| \lambda y^{\prime} z^{\prime} \cdot s_{2} \mid=\operatorname{makc}\left(\left.K\right|_{O_{1}}, y^{\prime}, z^{\prime}\right) \\
& \lambda x . s_{1}\left|=\operatorname{read}\left(x,\left.N\right|_{0_{2}},\left.B\right|_{0_{3}}\right) \quad \| \quad \lambda y^{\prime} z^{\prime} \cdot s_{2}\right|=\operatorname{makc}\left(\left.K\right|_{0_{4}}, y^{\prime}, z^{\prime}\right) \\
& \lambda x y . s_{1}\left|=\operatorname{read}(x, y, B) \quad \| \quad \lambda z^{\prime} \cdot s_{2}\right|=\operatorname{make}\left(\left.K\right|_{\theta_{1}},\left.N\right|_{\theta_{5}}, z^{\prime}\right) \\
& \| s_{2} \mid=\operatorname{make}\left(\left.K\right|_{\theta_{4}},\left.N\right|_{0_{5}}, \lambda y \cdot \operatorname{read}(N, y, B)\right)
\end{aligned}
$$

Figure 4: Process 


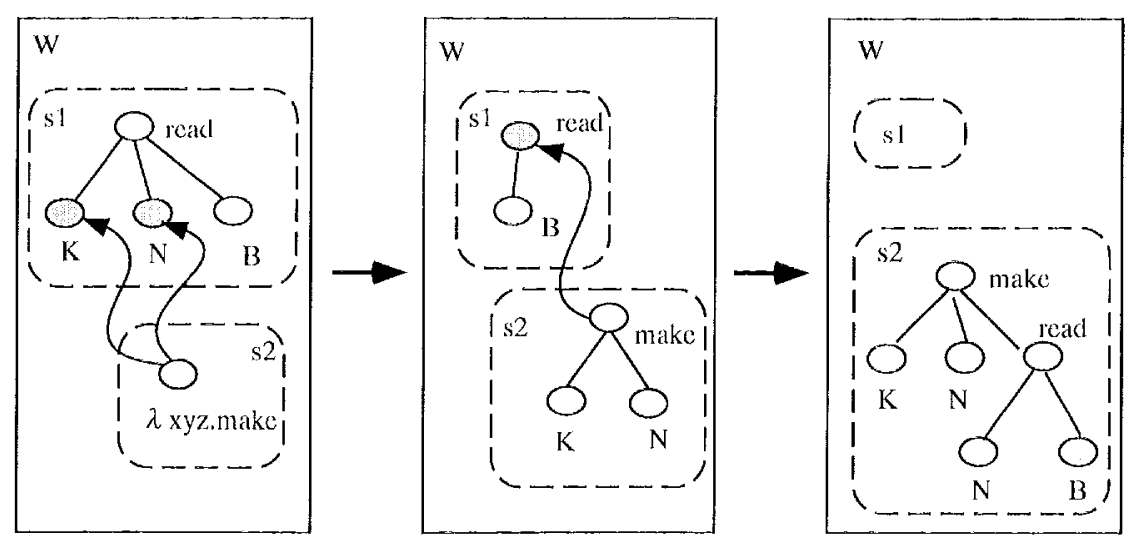

Figure 5: Membranes interaction

\section{Conclusion}

Introducing free-ordered typed $\lambda$-calculus, together with the notion of unification costs in lypes, we have shown the structuring of natural language syntax, by distributively represented types in random orders. We adopted a model of Chemical Abstract Machine for the partial/ concurrent computation model.

Nlthougl. we introduced the concept of costs and termination was assured, the efficiency of constructing a parsing tree would be far slower than secpuential processing. However onl objective is not to propose a faster algorithm, but is to show the possibility of clistributed processing of natural languages. We could show that natural language syntax is self-organizable, in that cach linguistic objectis do not need to be poured in to 'molds', viz., cxternally given gramenar.

\section{References}

[1] G. Berry and G. Bondol. 'L'ho chomical abstract machine. In $17 \mathrm{~h}$ Annual ACM Symposium on Principles of Programming Languages, pages $81.93,1990$.

[2] II. B. Curry and R, leys. Combinatory Logic, volume 1. North Holland, Amsterdan, Nethorlands, 1968.

[3] 1). Dowty. Type Raising, Functional Composition, and Non-constituont Conjunction - in Calegorial Grammars and Nalural Language. Structures, pages $153-197$. 1). Reidel, 1988.

[1] J. R. Mindloy and Soldin J. P'. Introduction to Combination and $\lambda$-Calculus. Cambridge University Pross, 1986.
[5] S. M. Shieber. An Introduction to Unification-Based Approaches lo Grammar. CSII, Stanford University, 1986.

[6] M. Steedman. Combinators and grammars - in Calegorial Grammars and Natural Tanguage Structures, pages 417-142. D. Reidel, 1988.

[7] S. Tojo. Categorial analysis of sentence generation. In The Iogic l'rogramming Conference (LPC 91), pages 229-238. Institule of New Generation Computer (ICO'T), 1991.

[8] H. Uszkoreit. Categorial unification grammars. In Proc. of COIING '86, pages 187$194,1.986$.

[9] 'I'. Gunji. Japanese Phrase Structure Grammar. J). Reidel, 1987. 